\title{
DESARROLLO DE UN ESCENARIO DE CAMPO PARA EL ESTUDIO DE ESPECIES NATIVAS DE ABEJORROS (Bombus spp.) DE LOS ANDES COLOMBIANOS (HYMENOPTERA: APIDAE)
}

Fecha de recepción: 9 de julio de 2013 • Fecha de aceptación: 15 de octubre de 2013

\author{
DEVELOPING OF A FIELD SCENARIO FOR THE STUDY OF NATIVE SPECIES OF \\ BUMBLEBEES (Bombus spp.) FROM THE COLOMBIAN ANDES (HYMENOPTERA: APIDAE) \\ Eddy Romero ${ }^{1}$ Carlos Pinilla Cruz ${ }^{2} \bullet$ José Ricardo Cure ${ }^{3}$ Diego Riaño ${ }^{4}$ Sandy Padilla ${ }^{2}$ Marlene Lucia Aguilar ${ }^{4}$
}

\section{RESUMEN}

El abejorro nativo Bombus atratus tiene una amplia distribución geográfica, está adaptado a diferentes condiciones ambientales y ha demostrado ser una especie muy eficiente en la polinización de diferentes cultivos. El grupo de Biodiversidad y Ecología de Abejas Silvestres (BEAS) ha venido desarrollando estudios básicos y aplicados para la utilización de esta especie y más recientemente se ha interesado en el desarrollo de metodologías para conseguir mejores resultados para su cría en mayor escala. En este momento se ha establecido un modelo experimental de cría, combinando las condiciones de campo e invernadero, donde, bajo prácticas agroecológicas, exista una oferta de alimento permanente, conservando una alta riqueza floral, abriendo la posibilidad de mantener allí simultáneamente otras especies nativas del género Bombus. Este es el concepto de Bombinario, que es un área física destinada a la cría de especies de Bombus, ubicada en el Campus de la Universidad Militar Nueva Granada en Cajicá. Está se proyecta como un área de investigación, docencia y proyección a la comunidad en temas de bionomía, ecología y comportamiento de los abejorros nativos, y de aspectos aplicados relacionados con la ecología de la polinización, manejo de los cultivos florales y cría de los abejorros. El proceso de desarrollo y creación del Bombinario es el objetivo de este trabajo.

Palabras clave: Bombus atratus, cría, riqueza floral, agroecología, polinización.

Técnico Asistencial de Laboratorio, Facultad de Ciencias Básicas y Aplicadas, Universidad Militar Nueva Granada.

2 Asistente de Investigación, Facultad de Ciencias Básicas y Aplicadas, Universidad Militar Nueva Granada.

3 Docente Programa Biología Aplicada, Facultad de Ciencias Básicas y Aplicadas, Universidad Militar Nueva Granada. Autor para correspondencia: jose.cure@unimilitar.edu.co.

4 Docente Programa Biología Aplicada, Facultad de Ciencias Básicas y Aplicadas, Universidad Militar Nueva Granada. 


\section{ABSTRACT}

The native bumblebee Bombus atratus has a broad geographical distribution, is well adapted to different environmental conditions and is a very good pollinator of a wide variety of crops. The Wild Bees Biodiversity and Ecology research group of UMNG (BEAS) has been interested in the study of the basic biology of this species and, more recently, in the development of mass rearing methodologies. At this point, the Group has developed an experimental rearing facility, combining green house and open field conditions, with a continuous supply of food, using agro ecological practices to maintain high floral richness, which opens the possibility to rear simultaneously different bumblebee species. This is the concept of the Bombinario, which is located at the Universidad Militar Nueva Granada Campus in Cajicá, Cundinamarca, Colombia. This area projects as a research facility for bionomic studios of bumblebees, and as community extension unit to illustrate the importance of pollination and characteristics of bee life. The process of building the Bombinario is here described.

Keywords: Bombus atratus, rearing, floral richness, agroecology, pollination.

\section{INTRODUCCIÓN}

A partir de la Cumbre de la Tierra, conferencia del medio ambiente realizada en Rio de Janeiro en el año 2000, surgió la Iniciativa Internacional sobre Polinizadores la cual ha contribuido al reconocimiento de la importancia de los organismos polinizadores para la conservación, tanto de los ecosistemas naturales como de los agroecosistemas. Esta iniciativa, a nivel global se ha venido consolidando en una serie de iniciativas regionales para la conservación de los polinizadores, por ejemplo, la europea (EPI, 2012; NAPPC, 2012), la africana (FAO, 2003a; NAPPC, 2012) la norteamericana (NAPPC, 2012), la brasileña (Días et al., 1999; NAPPC, 2012), la de Oceanía (NAPPC, 2012; OPI, 2012) y, más recientemente la colombiana (ICPA, 2010; NAPPC, 2012). También ha generado el proyecto mundial sobre polinización, centrado en la "Conservación y gestión de polinizadores para la agricultura sostenible a través de un enfoque "ecosistémico" de la FAO, el PNUMA y el FMAM (FAO, 2003b). La riqueza biológica del país solamente se incorporará al patrimonio cultural nacional en la medida que conozcamos lo que tenemos, abriendo nuevos horizontes para el desarrollo sostenible y utilización creativa de nuestras riquezas naturales.

La preocupación por la conservación de estos organismos es aún mayor hoy en día que cuando nació la Iniciativa Internacional hace 13 años, a raíz de la constatación del decaimiento y pérdida, en escala masiva, de las colonias de la abeja común, en varias partes del mundo, conocido como CCD (del Inglés colony-collapse disorder).

Las abejas, grupo biológico que a nivel mundial cuenta con más de 20.000 especies, son organismos especializados y totalmente dependientes de la recolección de polen y néctar para su sobrevivencia (Roubik, 1989). El grupo de investigación en Biodiversidad y Ecología de Abejas Silvestres (BEAS) ha venido estudiando desde hace más de diez años las especies de abejorros del género Bombus presentes en la Sabana de Bogotá y sus alrededores, 
particularmente en aspectos relacionados con la ecología y comportamiento de la especie nativa B. atratus. A partir del conocimiento adquirido se ha iniciado la cría de esta especie en condiciones controladas, con la finalidad de utilizarla en polinización de hortalizas de importancia económica (Aldana et al., 2007; Bernal et al., 2007; Morales, 2008; Torres y Ardila, 2010, Aguilar et al., 2010; Almanza, 2007; Chavarro, 2008; Zuluaga, 2011; Poveda et al., 2012; Pacateque et al., 2012; Camelo et al., 2004; Lobaton et al., 2012). Con la misma finalidad se han venido realizado estudios básicos en otras especies nativas de este género de abejas, tales como $B$. hortulanus y B. rubicundus. (Rubio, 2003; Álvarez, 2004; Aguilar, 2004).

En los primeros años la cría se desarrollaba en forma controlada en cámaras de cría, dependiendo en gran medida de reinas colectadas en campo en la sabana de Bogotá y demandando mano de obra para alimentación diaria de las colonias. Gracias a pruebas preliminares de cría externa (con acceso de los individuos de la colonia a los recursos florales del medio natural) realizadas en años anteriores y las inversiones realizadas por la universidad en la construcción de nuevos invernaderos, se abrió la posibilidad de estudios de campo más ambiciosos, dentro de los cuales sobresale la construcción de un área externa para el desarrollo de estudios sobre bionomía y ecología aplicada de los abejorros nativos de la Sabana de Bogotá y alrededores.

\section{MATERIALES Y MÉTODOS}

\section{Descripción del área}

La zona del Bombinario está conformada por una infraestructura de invernadero y un área externa bien delimitada, que conjuntamente suman aproximadamente $2000 \mathrm{~m}^{2}$ (Figuras 1 y 2), localizados en el interior del Campus Universitario de la Universidad Militar Nueva Granada, en Cajicá, Cundinamarca. Esta área tiene acceso a infraestructura de riego, a partir de un reservorio que atiende varios laboratorios de campo de la Facultad de Ciencias Básicas y Aplicadas. El Bombinaro tiene continuidad con áreas verdes que existen alrededor, incluyendo cultivos hortícolas, vegetación silvestre, árboles de eucaliptus y pasto kikuyo (Penissetum clandestinum). Estas áreas están delimitadas en su porción oriental por el río Bogotá, que forma allí un humedal; hacia el oriente es continua con extensos campos de golf. En su área occidental las áreas verdes están distribuidas entre las construcciones de las diversas facultades del Campus de la Universidad en Cajicá. El invernadero es cerrado por malla anti-trips en todo su perímetro y en la zona de cumbrera (apertura en la parte superior del invernadero).
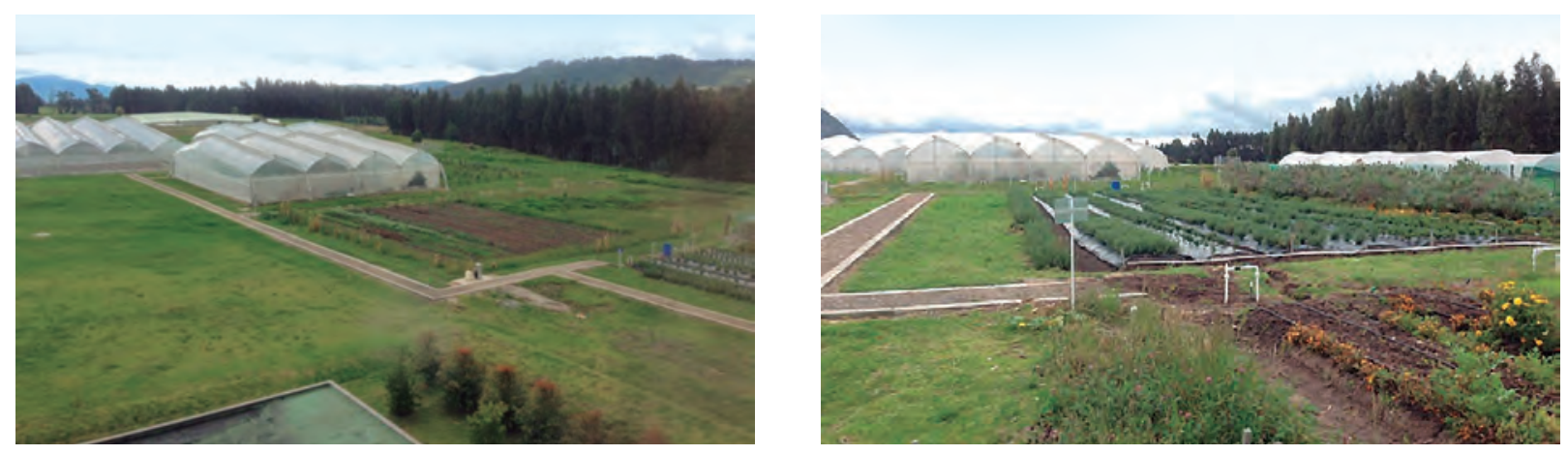

Figura 1. Localización del Bombinario en el Campus universitario de Cajicá. 


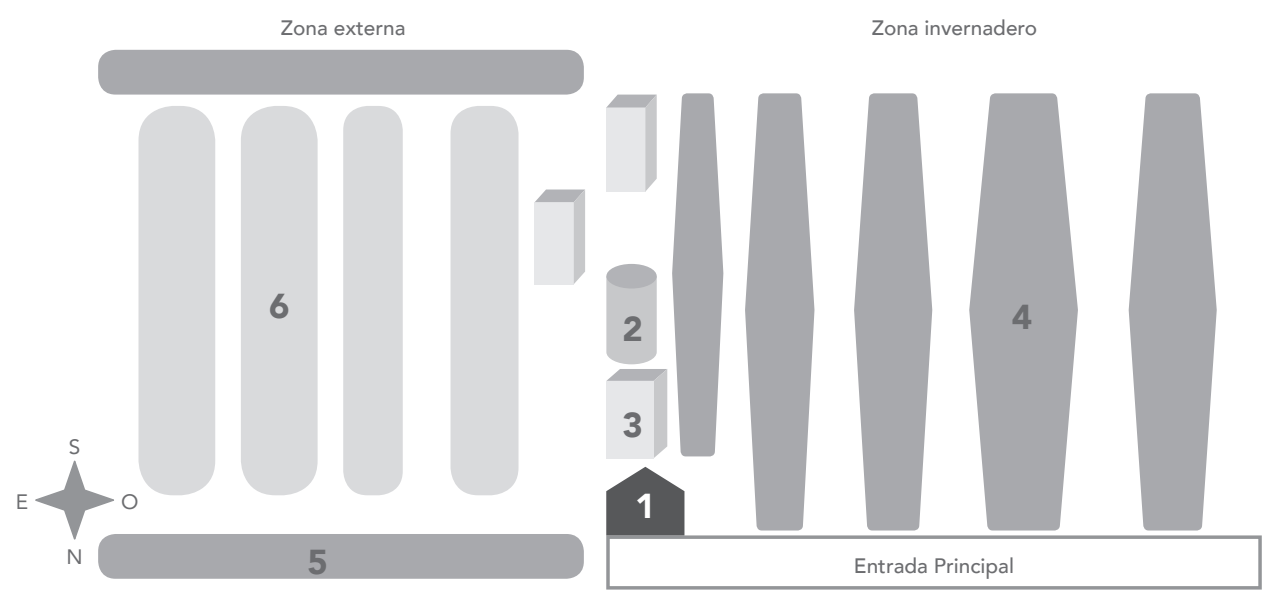

Figura 2. Esquema que ilustra la organización del Bombinario: 1) módulo de cría, 2) nidos trampa, 3) colonias, 4) camas de siembra, 5) cerca viva de mora (Rubus glaucus), 6) camas de siembra del exterior.

Las condiciones del suelo para cultivo de plantas en el área del Bombinario son limitantes para el crecimiento y desarrollo vegetal, el suelo es arcilloso, de bajo contenido en materia orgánica y estructura muy pobre. La capa fértil (horizonte A) se había removido en el proceso de urbanización del Campus.

Se establecieron desde un inicio algunas premisas básicas para orientar el desarrollo del Bombinario: a) es un espacio donde se agrupa una muestra de la rica diversidad florística de la zona, en sus diferentes estratos de vegetación, teniéndose como ideales aquellas especies perennes de fácil establecimiento y manejo, que en lo posible, no exijan prácticas culturales intensivas para su establecimiento y mantenimiento y que provean una amplia y abundante fuente de néctar y polen para las abejas; b) es un área en donde se debe utilizar una baja cantidad de fertilizantes de síntesis química y eliminar completamente la utilización de pesticidas; c) debe tenerse en cuenta una visión de sostenibilidad dentro de una perspectiva agroecológica para la regulación de las plagas potenciales, utilización de abonos orgánicos, cultivos de cobertura, alelopatía, labranza mínima entre otros, siempre en procura de evitar el impacto antrópico en el medio y darle a la zona una estabilidad en el tiempo; d) la distribución y composición florística del área y la disposición de las zonas de cría para los nidos se orienta a generar un espacio en el que las interacciones entre plantas y abejorros se ocurran de forma espontánea y natural, donde sea posible realizar observaciones y estudios de biología básica de las especies, su comportamiento y las relaciones entre ellas; e) esta área será un espacio propicio para la práctica de la permacultura, entendida como una estrategia armónica donde se logre una interacción entre el ser humano y el ambiente, donde se pueda desarrollar una unidad demostrativa, didáctica, para la enseñanza de temas de polinización, basada en el concepto de productividad, en base a los recursos disponibles, bajo un manejo con poca utilización de insumos, cobertura verde y labranza mínima, dentro de un concepto de manejo ambiental.

Se partió de una lista de especies en base a experiencias realizadas por los autores, las cuales fueron un insumo inicial para los primeros desarrollos del Bombinario. Las áreas disponibles se distribuyeron de acuerdo a lo indicado en la Fig. 2.

\section{Origen de las primeras colonias}

Las colonias iniciales provenían de una cría en laboratorio en ambiente controlado de temperatura y 
humedad, a partir de reinas silvestres recolectadas en varios puntos de la Sabana de Bogotá. Posteriormente, cuando tenían más de 30 obreras las colonias se trasladaron a campo abierto, esperando las condiciones adecuadas para su instalación definitiva en el Bombinario.

\section{RESULTADOS}

Primera fase: Acondicionamiento de las áreas de siembra

El proceso de acondicionamiento del terreno, tanto en el área externa como en la de invernadero, se inició a finales del mes de julio de 2012, descompactando el suelo manualmente e incorporando varios metros cúbicos de tierra fértil proveniente de otras áreas del Campus de Cajicá (Fig. 3a). Durante ese período se permitió el crecimiento espontáneo de arvenses y simultáneamente se inició la siembra de 27 plantas de lulo (Solanum quitoense), teniendo en cuenta que en experiencias anteriores mostró ser una planta muy frecuentada por los abejorros, se adapta a las condiciones del invernadero y provee permanentemente abundante polen. El lulo se sembró en tres surcos, con densidad de 1 planta por $5 \mathrm{~m}^{2}$, dejando espacio suficiente entre ellas para sembrar otras especies y así proveer recursos adicionales y variados para las colonias. Esta siembra se complementó con un surco de papa con la finalidad de ofrecer rápidamente mayor oferta de polen (Fig. 3b).

Una cubierta vegetal consistente en heno de leguminosas y kikuyo (Pennisetum clandestinum) fue utilizada para proteger el suelo, evitando la pérdida de agua, la radiación directa y la competencia con otras plantas. Más o menos de forma simultánea a lo anterior se distribuyeron, al voleo, semillas de rábano (Raphanus sativus) y colza (Brassica napus), plantas anuales de ciclo corto, sistema radicular profundo, gran producción de biomasa por hectárea y apreciable producción de néctar y polen al poco tiempo de la siembra (45-60 días). El rábano germinó primero, a comienzos del mes de agosto (Fig. 3c) y un mes después ya se presentaba una gran masa vegetal que cubría toda el área, habiendo florecido hacia el día 18 de septiembre (Fig. 3e).

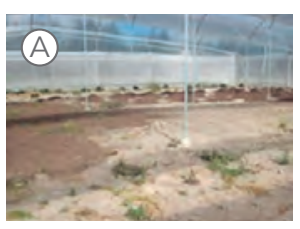

$25 / 7 / 2012$

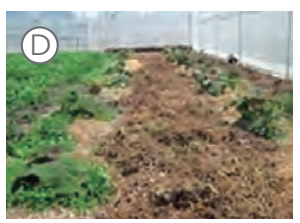

$7 / 9 / 2012$

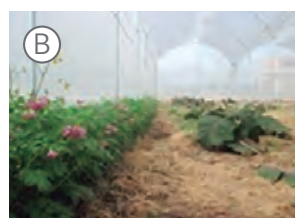

$6 / 8 / 2012$

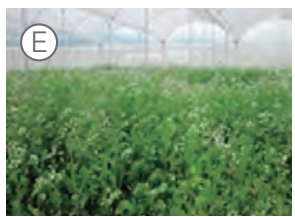

$18 / 9 / 2012$

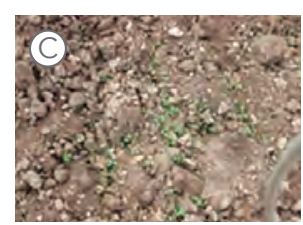

$6 / 8 / 2012$

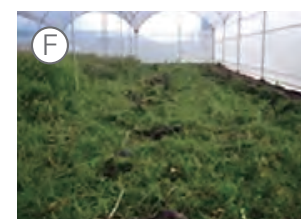

$18 / 10 / 2012$

Figura 3. Fotografías que muestran el proceso de adecuación del Bombinario. 
Una vez el suelo se encontraba cubierto totalmente por plantas arvenses, gran parte de estas fueron podadas y utilizadas como cobertura vegetal para el suelo (Figura 3f). Sobre esa cobertura se sembraron más plantas de lulo, así como otras plantas de ciclo corto tales como el girasol (Helianthus annuus) y tomate (Solanum lycopersicum).

En el área externa del Bombinario se realizó siembra al voleo de carretón morado (Trifolium pratense) como cultivo de cobertura, junto con algunas plantas de lulo y de lupinus (Lupinus mutabilis).

\section{Segunda fase: Selección y propagación de especies vegetales}

Las especies vegetales fueron seleccionadas con base en observaciones previas y trabajos o reportes realizados por el grupo de Biodiversidad y Ecología de Abejas Silvestres (Tabla 1). Se incluyeron tanto especies anuales como perennes, buscando mantener un suministro constante de polen y néctar para las colonias de abejorros.

En la Tabla 2 se presenta una aproximación de la contribución de las diferentes especies de plantas como recursos de polen o néctar a lo largo del desarrollo del Bombinario. Sobresalen el lulo y el girasol, por su importancia como fuentes de polen abundante y fácil adaptación a las condiciones del suelo.

La propagación de las plantas en su mayoría se ha realizado por semillas, las cuales se mantienen 24 horas antes de su siembra en bandeja de germinación en proceso de imbibición (pre germinación) de manera que el proceso de germinación sea más corto. Las plantas de botón de oro y, en algunos casos también las de lulo, son propagadas por esquejes.

Tercera fase: introducción de las colonias

La floración del lulo, la papa, el rábano y de la colza, dentro del invernadero y la presencia de carretón morado y carretón blanco blanco en el exterior, así como algunas otras plantas herbáceas espontáneas,
Tabla 1. Plantas seleccionadas como fuente de alimento para las colonias de $B$. atratus en el Bombinario.

\begin{tabular}{|c|c|c|}
\hline $\begin{array}{l}\text { Nombre } \\
\text { común }\end{array}$ & $\begin{array}{l}\text { Nombre } \\
\text { científico }\end{array}$ & $\begin{array}{l}\text { Recurso } \\
\text { ofrecido }\end{array}$ \\
\hline Arándano azul & $\begin{array}{l}\text { Vaccinium } \\
\text { corymbosum }\end{array}$ & Polen y Néctar \\
\hline Berenjena & $\begin{array}{c}\text { Solanum } \\
\text { melanogena }\end{array}$ & Polen \\
\hline Borraja & Borago officinalis & Néctar \\
\hline Botón de oro & Tithonia diversifolia & Néctar \\
\hline Calabacín & Cucurbita pepo & \\
\hline Cardo & Cardos acartoides & Polen \\
\hline Carretón blanco & Trifolium repens & Polen y Néctar \\
\hline $\begin{array}{l}\text { Carretón } \\
\text { morado }\end{array}$ & Trifolium pratense & Polen y Néctar \\
\hline Cocona & $\begin{array}{c}\text { Solanum } \\
\text { sessiliflorum }\end{array}$ & Polen \\
\hline Colza & Brassica nappus & Néctar \\
\hline Dalia & Dahlia sp & Néctar \\
\hline Diente de león & $\begin{array}{l}\text { Taraxacum } \\
\text { officinale }\end{array}$ & \\
\hline Girasol & Helianthus annuus & Polen \\
\hline Guava & $\begin{array}{l}\text { Phytolacca } \\
\text { bogotensis }\end{array}$ & \\
\hline Lulo & Solanum quitoense & Polen \\
\hline Lupino & Lupinus mutabilis & Polen \\
\hline Maíz & Zea mays & Polen \\
\hline Malva & Lavatera arbórea & Polen y Néctar \\
\hline Manzanilla & $\begin{array}{c}\text { Chamaemelum } \\
\text { nobile }\end{array}$ & Polen \\
\hline Nabo forrajero & Raphanus sativus & Néctar \\
\hline Oxalis amarillo & Oxalis stricta & \\
\hline \multirow[t]{2}{*}{ Romero } & $\begin{array}{c}\text { Rosmarinus } \\
\text { officinalis }\end{array}$ & Polen \\
\hline & $\begin{array}{c}\text { Solanum } \\
\text { lycopersicum }\end{array}$ & Polen \\
\hline Tomate & $\begin{array}{c}\text { Polygonum } \\
\text { persicaria }\end{array}$ & Polen \\
\hline
\end{tabular}

ISSN 1900-4699 • Volumen 9 • Número 2 • Páginas 200-211 • 2013 
Tabla 2. Principales plantas utilizadas a lo largo del tiempo como fuentes de polen o néctar en el invernadero a partir del inicio del Bombinario ( $\mathrm{P}=$ pólen, $\mathrm{N}=$ néctar).

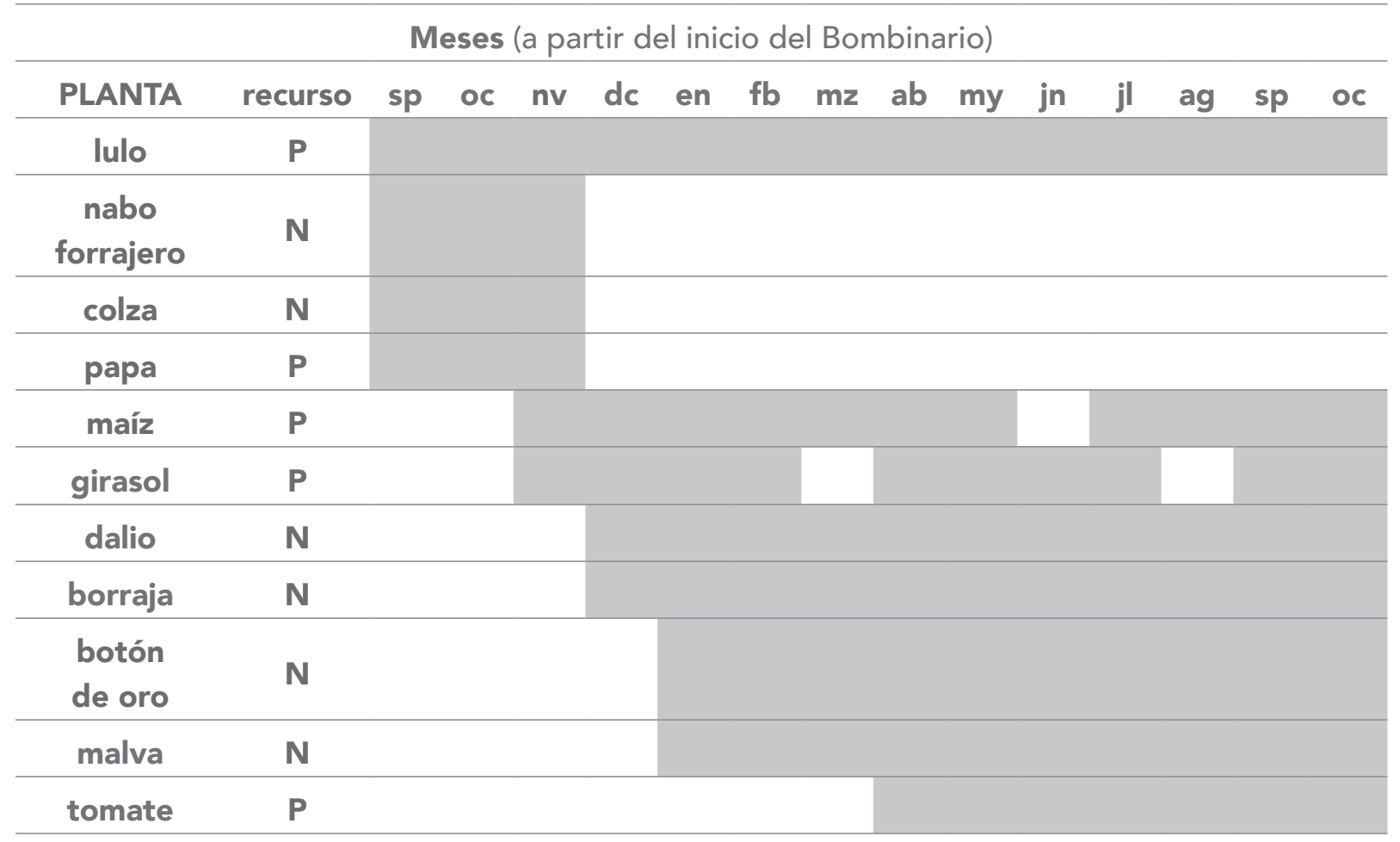

permitió el traslado de las primeras colonias de Bombus al Bombinario en el mes de septiembre. Siendo el invernadero cerrado por malla, las colonias se instalaron con dos tubos de comunicación (piqueras), uno que permitía el acceso al interior del invernadero y otro que comunicaba con el área externa, lo que aumentaba la oferta y diversidad del recurso floral para las colonias (Fig.4). Se continuó con la siembra de nuevas especies, como el girasol, botón de oro y dalio, las cuales mostraron gran adaptación a las condiciones medio ambientales.

Cuarta fase: establecimiento del módulo de cría

El Bombinario se convirtió en un lugar apropiado para mantener las colonias, puesto que estas mostraban un buen crecimiento y podían ser observadas obreras, reinas y machos todo el tiempo visitando las flores e interactuando con las plantas.

Surgió la idea de un lugar dentro del invernadero en donde se pudieran mantener colonias de la misma manera como se venía realizando en este lugar pero a su vez nos permitiera realizar observaciones del interior de las colonias sin que estas sufran algún tipo de estrés por la incidencia de luz y por la presencia de los observadores. Para ello se construyó el módulo de cría, el cual sería a largo plazo el reemplazo de la cámara de cría en el laboratorio; este debería ser un lugar oscuro y que tuviera las comodidades necesarias para realizar buenas observaciones de las colonias. 


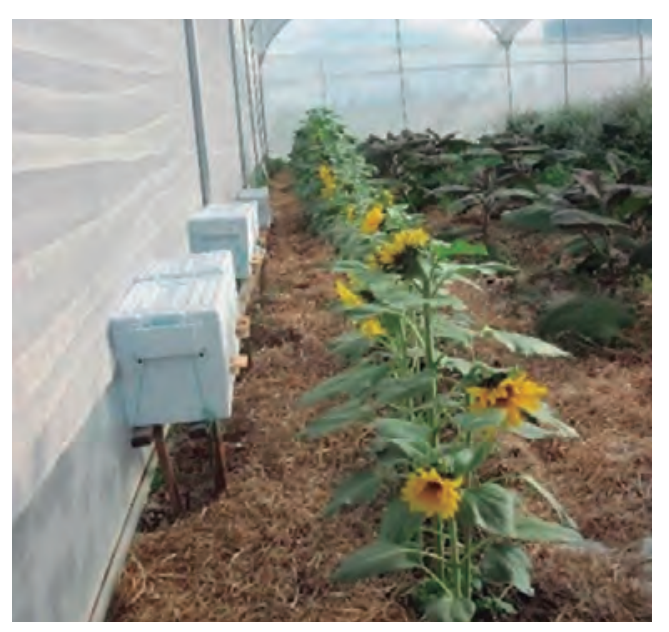

Figura 4. Disposición de las colonias en el invernadero

El módulo de cría consiste en una estructura con bases metálicas, recubierta con 3 capas de polisombra negra (80\%) y puerta en madera triplex. Esta estructura posee medidas de $2.20 \mathrm{~m}$ de alto, 3 $\mathrm{m}$ de largo y $1.50 \mathrm{~m}$ de ancho, tiene el objetivo de mantener colonias de abejorros, desde sus etapas tempranas de desarrollo, sustituyendo la cámara de cría en laboratorio, lugar donde se venía llevando a cabo la cría de las colonias (Fig. 5). Dentro de esta estructura se acondicionaron estantes sobre los cuales se soportan las colonias contenidas en cajas de poliestireno expandido acondicionadas para tal fin.

Una de las novedades y mejoras con respecto a la cámara en laboratorio es la posibilidad de que las colonias que se encuentran en el módulo tengan salida hacia el interior o el exterior del Bombinario, lo que permite que las obreras se encarguen de buscar su propio alimento, permitiendo una mayor eficiencia en la alimentación de las colonias. Al interior del módulo de cría, en la situación actual, se presenta temperatura promedio de $16.98^{\circ} \mathrm{C}$ con máximas de $28.6^{\circ} \mathrm{C}$, mínimas de $8.58{ }^{\circ} \mathrm{C}$ y humedad relativa de $69.62 \%$. Próximas mejoras deberán realizarse para conseguir condiciones internas más
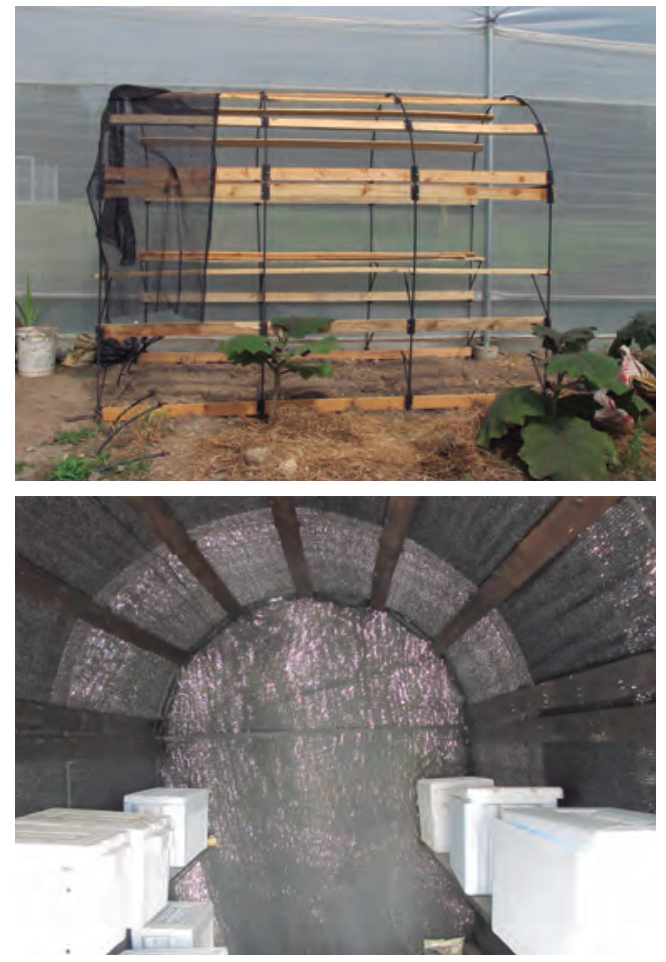

Figura 5. Imagen del módulo de cría donde se encuentran actualmente algunas colonias.

estables dentro de la cámara. Una ventaja que ya es posible valorar es la disminución del esfuerzo y costos relacionados con la alimentación manual de las colonias cuando la iniciación de las mismas se realiza en condiciones de laboratorio.

\section{Quinta fase: estabilización y manejo}

La cobertura vegetal cumple una función primordial dentro del esquema del Bombinario, evitando la pérdida de humedad y nutrientes y además sirve para controlar la temperatura y erosión del suelo (Fig.6). Asimismo, esta cobertura evita la proliferación de plantas arvenses y es un refugio para muchas especies de insectos benéficos (parasitoides y depredadores y ofrece sustrato para que algunas especies de abejas y abejorros construyan sus nidos. 
Han sido utilizado macrófitas flotantes tomadas de los reservorios de agua del Campus universitario, como un abono orgánico y cobertura del suelo, este brinda cantidades importantes de nitrógeno y otros nutrientes. Del mismo modo, se han venido utilizando restos de plantas de frijol, pasto seco y los restos de plantas producidos en el Bombinario como cobertura vegetal.

A pesar de que muchas de las especies vegetales seleccionadas no requieren de un mantenimiento en sentido estricto, es necesario realizar algunas labores culturales que permitan su buen desarrollo y crecimiento. La adecuación, mantenimiento y producción de plantas se han realizado en base a la utilización de prácticas agroecológicas, teniendo en cuenta la necesidad de mantener una heterogeneidad de plantas, con diferentes susceptibilidades a lo herbívoros, de tal forma que haya un equilibrio ente las posibles plagas de las plantas sembradas allí y sus enemigos naturales. Prácticas de mantenimiento vegetal como las podas las plantas de tomate, el deschuponado a plantas de lulo y tomate, el aporcado a las plantas de maíz y el tutorado a las plantas de tomate son realizadas periódicamente.

En la medida que se ha venido incrementando el número de colonias en el Bombinario, ha sido necesario adicionar nuevas especies vegetales y hacer un mejoramiento en la adecuación de las camas, antes

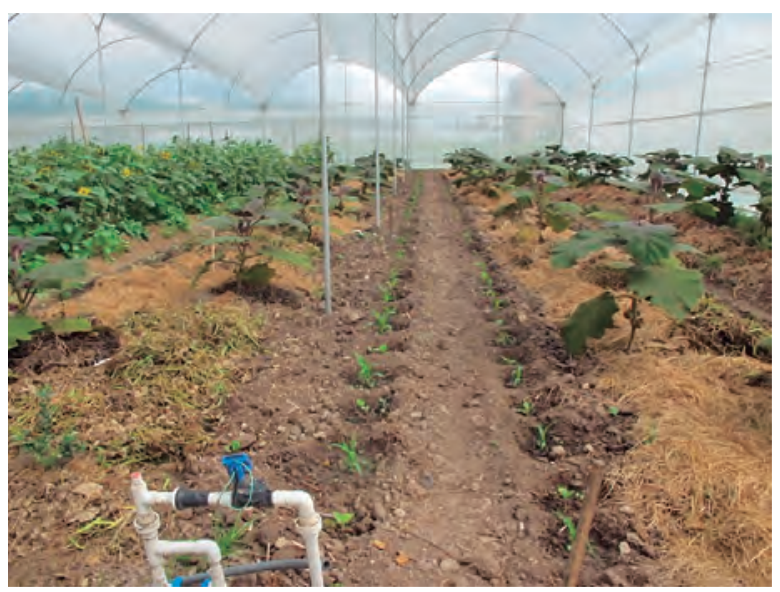

Figura 6. Algunas camas de siembra y cobertura vegetal utilizada en el Bombinario.

de la siembra para permitir su mejor crecimiento. Dentro de esta adecuación se realizó una adición de tierra, la cual se mezcló con el suelo ya existente, en los cuales se construyeron surcos a los que se adicionaba carbón y gallinaza, se mezclaba nuevamente con el suelo existente, e inmediatamente se regaba la cama completa. Después de que las camas fueron sembradas se colocó sobre ellas la cobertura vegetal. En el área externa del Bombinario también fue necesario preparar las camas de una mejor manera, se siguieron sembrando plantas de lulo y se dio gran importancia a la conservación de arvenses, que han demostrado ser una buena fuente de alimento para

\section{A pesar de que muchas de las especies vegetales seleccionadas no requieren de un mantenimiento en sentido estricto, es necesario realizar algunas labores culturales que permitan su buen desarrollo y crecimiento.}


las colonias. Se espera continuar con el proceso de evaluación de nuevas especies vegetales útiles para su establecimiento dentro del Bombinario y de esta manera tener una gran variedad de fuentes de alimento para las especies del genero Bombus presentes en este espacio y a su vez grandes oportunidades en la investigación de interacciones ecológicas entre planta-abejorro.

Dentro de los propósitos a corto plazo se buscan adecuar senderos para facilitar procesos de enseñanza-aprendizaje, permitiendo un acercamiento entre la comunidad, la cría, y la conservación de estas especies y asimismo aumentar el interés en el estudio de los abejorros y su importancia dentro del ecosistema.

\section{DISCUSIÓN}

Debido a la reducción poblacional de varias especies de polinizadores, reportada en las últimas dos décadas, en todo el mundo, es de gran importancia el diseño de estrategias para su conservación. Entre los polinizadores más eficientes, que han sufrido también el impacto anteriormente mencionado, encontramos el género Bombus (Goulson et al., 2008; Grixti et al., 2009), que incluye importantes especies polinizadoras de numerosas especies de plantas, entre las cuales sobresalen las solanáceas. Entre las especies de Bombus que venimos trabajando, B.atratus, por ejemplo, es una especie de amplia distribución, muy adaptable a condiciones climáticas variables y a vegetación muy diversa. Ha sido estudiada como un buen candidato para la polinización dirigida de plantas de interés comercial, tales como tomate, lulo, carretón, etc. (Almanza, 2007; Aldana et al., 2007; Lobaton et al., 2012).

Una de las formas de contribuir a la conservación de las especies de este género es adquirir mayor conocimiento sobre su bionomía y requerimientos ecológicos, y desde el punto de vista de su utilización, es necesario desarrollar técnicas para su reproducción en condiciones controladas, de tal forma que se pueda ofrecer esta alternativa como una solución viable para la polinización de frutas y hortalizas. Ejemplos de conservación, en donde además de investigación se puedan tener unidades demostrativas para llevar a la comunidad conocimiento sobre la importancia de estos organismos y su interacción con las plantas, son muy oportunos y, como en el caso del Bombinario aquí descrito, se vienen desarrollando iniciativas en este sentido en otras partes del mundo como por ejemplo en la Universidad de California en Davis (http://beebiology.ucdavis.edu/ HAVEN/index.html), o en Berkeley (http://www.helpabee.org/), Estados Unidos, o como el "Bee Garden" de Málaga, España, con colmenas móviles en una estructura de cristal, donde se puede observar toda la actividad de las abejas (http://beegardenmalaga.com/about/ ). 


\section{BIBLIOGRAFÍA}

1. Aguilar M. 2004. Biología y nidación de Bombus rubicundus Smith, 1854 (Hymenoptera: Apidae) en condiciones de cautiverio. Tesis de grado. Universidad Militar Nueva Granada.

2. Aguilar M, Cantor F, Cure J.R, Rodríguez D, Bajonero J, Pérez D, Riaño D. 2010. Integración de conocimientos y tecnologías de polinización y control biológico. Universidad Militar Nueva Granada. ISBN 978-958-8403-27-4.

3. Aldana J, Cure J, Almanza M, Vecil D, Rodríguez D. 2007. Efecto de Bombus atratus ( $\mathrm{Hy}$ menoptera: Apidae) sobre la productividad de tomate (Lycopersicon esculentum Mill) bajo invernadero en la sabana de Bogotá, Colombia. Agronomía colombiana. 25(1): 62-71.

4. Almanza M. 2007. Management of Bombus atratus bumblebees to pollinate lulo (Solanum quiotense L), a native fruit from the Andes of Colombia. ZEF. Ecology and Development Series.

5. Álvarez C. 2004. Iniciación de colonias de abejorros de la especie Bombus hortulanus (Friese) (Hymenoptera: Apidae) en cautiverio. Trabajo de grado. Universidad Militar Nueva Granada. Colombia.

6. Bernal S, Cure J. R, Rodríguez D, Pérez M.M, Almanza M.T. 2007. Oferta Floral y polinización de tomate bajo invernadero con Bombus atratus Franklin (Hymenoptera: Apidae). Revista Facultad De Ciencias Básicas 3 (1): 91 - 100.

7. Camelo E., Díaz L., Cure J.R., Almanza M.T. 2004. Morfología floral de la uchuva y comportamiento de visitas de la especie de abejorros Bombus atratus (Hymenoptera: Apidae) bajo invernadero. Memorias XXI congreso de la
Sociedad Colombiana de Entomologia - SOCOLEN. Bogotá Colombia. 28 al 30 de julio.

8. Chavarro N. 2008. Determinación de la biología floral del Lulo (Solanum quitoense Lam.) y del efecto de la polinización con abejorros Bombus atratus sobre la producción. Tesis de maestría. Universidad Militar nueva Granada.

9. Días B, Anthony R, Vera L. 1999. Report on the Recommendations of the Workshop on the Conservation and Sustainable Use of Pollinators in Agriculture with Emphasis on Bee. The São Paulo declaration on pollinators. 72 pages.

10. FAO. 2003a. The Plan of Action of the African Pollinator Initiative. African pollinator initiative secretariat. 32 pages.

11. FAO, 2003b. Biodiversidad para un mundo sin hambre. http://www.fao.org/biodiversity/ componentes/polinizadores/es/. Accedida el 4/12/2013.

12. ICPA. 2010. Iniciativa colombiana de polinizadores. http://www.herbogeminis.com/IMG/ pdf/iniciativa_colombiana_de_polinizadores. pdf. Accedida el 11/09/2013.

13. Goulson D, Darvill B, Lye G. 2008. The decline and conservation of Bumblebees. Annual Review of Entomology. 53: 191-208.

14. Grixti J, Wong L, Cameron S, Favret C. 2009. Decline of Bumble bees (Bombus) in the North American Midwest. Biological conservation. 142: 75-84.

15. Lobaton J, Cure J, Almanza M. 2012. Fenología y oferta floral de trébol rojo Trifolium pratense (Fabales: Fabaceae) en praderas de kikuyo Penissetum clandestinum (Poales: Poaceae), 
como fuente alimento para Bombus atratus (Hymnoptera, Apoidea) en Cajicá, Colombia. Revista facultad de ciencias básicas. 8(1): 18-27.

16. NAPPC. 2012. International pollinator initiatives. http://pollinator.org/nappc/international. htm\#europe. Accedida el 11/09/2013.

17. OPI. 2012. About the Oceania Pollinator Initiative. http://www.oceaniapollinator.org/about. asp. Accedida el 11/09/2013.

18. Pacateque J., Riaño D., Aguilar L., Cure J.R. 2012. Comportamiento de forrajeo de Bombus atratus sobre el cultivo de pimentón (Capsicum annum) var Robledo bajo invernadero. Memorias X Encontro Sobre Abelhas. Ribeirao Preto. Brasil.25 - 28 de Júlio.

19. Poveda C., Aguilar M., Riaño D., Perez M.M. 2012. Comportamiento de polinización del abejorro nativo Bombus atratus (Hymenoptera: Apidae) en flores de dos cultivares de fresa (Fragaria $\times$ ananassa) y su efecto en el desarrollo de los frutos. Memorias X Encontro Sobre Abelhas. Ribeirao Preto. Brasil. 25-28 de Júlio.

20. Rubio D. 2003. Biología de nidación de Bombus hortulanus Friese, 1904 (Hymenoptera: APIDAE). Tesis de grado. Universidad Militar Nueva Granada.

21. Torres G. y Ardila J. 2010. Evaluación de la Actividad Polinizadora de Bombus atratus en cultivos de tomate bajo invernadero en Sachica y Villa de Leyva, Boyacá, Colombia. Tesis de grado. Universidad Militar Nueva Granada.

22. Roubik W. 1989. Ecology and natural history of tropical bees. Cambridge University Press, New York. 520 págs. 